\title{
Prevalence of oropharyngeal beta-lactamase-producing Capnocytophaga spp. in pediatric oncology patients over a ten-year period
}

\author{
Anne Jolivet-Gougeon*1, Zohreh Tamanai-Shacoori ${ }^{1}$, Laurent Desbordes ${ }^{1}$, \\ Virginie Gandemer ${ }^{2}$, Jean-Louis Sixou ${ }^{1}$, Nolwenn Morvan-Graveline ${ }^{1}$, \\ Michel Cormier ${ }^{1}$ and Martine Bonnaure-Mallet ${ }^{1}$
}

Address: ${ }^{1}$ Equipe de Microbiologie, UPRES-EA 1254, Faculté des Sciences Pharmaceutiques et Biologiques, Université de Rennes 1, 2 avenue du Professeur Léon Bernard, 35043 Rennes, France and 2Pediatric Oncology Department, CHU Hôpital Sud, 16 boulevard de Bulgarie, 35000 Rennes, France

Email: Anne Jolivet-Gougeon* - anne.gougeon@univ-rennes1.fr; Zohreh Tamanai-Shacoori - zohreh.shacoori@univ-rennes1.fr; Laurent Desbordes - desbordes@nomade.fr; Virginie Gandemer - virginie.gandemer@chu-rennes.fr; Jean-Louis Sixou - jean-louis.sixou@univrennes1.fr; Nolwenn Morvan-Graveline - g.graveline@free.fr; Michel Cormier - michel.cormier@univ-rennes1.fr; Martine BonnaureMallet - martine.bonnaure-mallet@univ-rennes1.fr

* Corresponding author

Published: 09 May 2005

BMC Infectious Diseases 2005, 5:32 doi:10.1 186/147/-2334-5-32

This article is available from: http://www.biomedcentral.com/I47I-2334/5/32

(C) 2005 Jolivet-Gougeon et al; licensee BioMed Central Ltd.

This is an Open Access article distributed under the terms of the Creative Commons Attribution License (http://creativecommons.org/licenses/by/2.0), which permits unrestricted use, distribution, and reproduction in any medium, provided the original work is properly cited.
Received: 05 November 2004

Accepted: 09 May 2005

\begin{abstract}
Background: The aim of this study was to evaluate the prevalence of beta-lactamase-producing Capnocytophaga isolates in young children hospitalized in the Pediatric Oncology Department of Hôpital Sud (Rennes, France) over a ten-year period (1993-2002).

Methods: In neutropenic children, a periodic survey of the oral cavity allows a predictive evaluation of the risk of systemic infections by Capnocytophaga spp. In 449 children with cancer, 3,053 samples were collected by oral swabbing and plated on TBBP agar. The susceptibility of Capnocytophaga isolates to five beta-lactams was determined.

Results: A total of 440 strains of Capnocytophaga spp. were isolated, 309 (70\%) of which were beta-lactamase producers. The beta-lactamase-producing strains were all resistant to cefazolin, $86 \%$ to amoxicillin, and $63 \%$ to ceftazidime. The proportion of strains resistant to third-generation cephalosporins remained high throughout the ten-year study, while susceptibility to imipenem and amoxicillin combined with clavulanic acid was always conserved.

Conclusion: These results highlight the risk of antibiotic failure in Capnocytophaga infections and the importance of monitoring immunosuppressed patients and testing for antibiotic susceptibility and beta-lactamase production.
\end{abstract}

\section{Background}

Capnocytophaga spp. are capnophilic, gram-negative fusiform rods with gliding motility, common inhabitants of the oral cavity, but their role as an etiologic agent in juve- nile periodontitis remains controversial [1]. In immunocompromised granulocytopenic patients, a number of complications, including septicemia, endocarditis, and peripheric lesions, have been reported [2-4]. Several 
authors have described cases of infections by Capnocytophaga strains resistant to antimicrobial agents [5-8]. An episode of bacteremia can be the consequence of bacterial translocation from oral flora. In the absence of bacterial strain isolation from blood cultures, empiric treatment might be adjusted according to the susceptibility of strains isolated during the survey. A sequential survey of the oral cavity during hospitalization was performed to evaluate the prevalence of beta-lactamase-producing Capnocytophaga isolates in children hospitalized in the Pediatric Oncology Department of Hôpital Sud (Rennes, France) over a ten-year period (1993-2002).

\section{Methods}

Samples were collected by swabbing the bucco-pharyngeal area of children hospitalized in the Pediatric Oncology Department of Hôpital Sud (Rennes, France) [9]. Samples were taken periodically with a minimum of 15 days between each collection. All cancer patients from one to 17 years were included whatever the type of cancer, chemotherapy, and clinical situation were. The number of samples taken per child depended on the oncological disease (Acute Lymphoblastic Leukemia, Acute Myeloblastic Leukemia, others), the number of cures, and the number and length of hospitalizations or treatments. Each sample was inoculated on TBBP agar plates [ $4 \%$ trypticase soy agar supplemented with $5 \%$ sheep blood, $0.1 \%$ yeast extract (AES Laboratory, France), $100 \mu \mathrm{g} / \mathrm{ml}$ polymyxin and $50 \mu \mathrm{g} / \mathrm{ml}$ bacitracin (Sigma)] [10], which was then incubated for two to five days in a $10 \% \mathrm{CO}_{2}$ atmosphere. Isolates were identified on the basis of colony morphology, Gram staining, negative catalase and oxidase reactions, API ZYM (BioMérieux, France) [11], and fatty acid profiles (gas chromatography, SHERLOCK Microbial Identification System $^{\text {TM }}$ MIDI Inc., Newark, DE, USA). Beta-lactamase production was tested using the qualitative chromogenic cephalosporin disk test ${\text { (Cefinase }{ }^{\circledast}, \mathrm{BBL}}$ Microbiology Systems, Cockeysville, MD, USA). The results were read after 30 minutes.

Susceptibility testing was determined by standard methods and break points using the criteria of Bremmelgaard et al. [12] for screening determinations, and NCCLS [13] for intermediate/resistant strains. Minimal Inhibitory Concentrations (MICs) were confirmed by the E-test method (AES Laboratory, Combourg, France) using the same incubation conditions. Researchers tested the following antibiotics: amoxicillin, amoxicillin combined to clavulanic acid, cefazolin, ceftazidime, and imipenem.

\section{Results}

Over the ten-year period of this study, researchers analyzed 3,053 samples from 449 hospitalized children (266 males and 183 females). Capnocytophaga spp (440 strains) were isolated in 232 children, on TBBP agar and identified with conventional methods. The annual percentage of children, who carried a Capnocytophaga strain at least once varied, with a minimum from 1995 to 1997 (17\% and 22\%, respectively), and maxima in 1993 (52\%), 2001 (58\%), and 2002 (61\%). These results were also observed studying the number of Capnocytophaga isolates in the same periods: the number of Capnocytophaga isolates changed, with a minimum $(11,6 \%)$ in 1996 and a maximum $(96,22 \%)$ in 2001 , without modification to the isolation and culture techniques (Table 1).

Table I: Prevalence of Capnocytophaga spp. strains in periodic oral samples from children hospitalized in the Pediatric Oncology Department of Hôpital Sud (Rennes, France) from 1993 to 2002

\begin{tabular}{lcccc}
\hline Year & $\begin{array}{c}\text { Number of children } \\
\text { included }\end{array}$ & $\begin{array}{c}\text { Number of Capno' } \\
\text { carriers } \text { (\%) }^{\prime}\end{array}$ & $\begin{array}{c}\text { Number of samples } \\
\text { collected }\end{array}$ & $\begin{array}{c}\text { Number of Capno iso- } \\
\text { lates (\%) }\end{array}$ \\
\hline 1993 & 60 & $31(52)$ & 305 & $56(18)$ \\
1994 & 50 & $24(48)$ & 228 & $49(21)$ \\
1995 & 45 & $10(22)$ & 195 & $14(7)$ \\
1996 & 49 & $8(16)$ & 198 & $11(6)$ \\
1997 & 58 & $9(17)$ & 251 & $13(5)$ \\
1998 & 62 & $24(39)$ & 296 & $41(14)$ \\
1999 & 68 & $24(35)$ & 267 & $38(14)$ \\
2000 & 84 & $17(20)$ & 347 & $31(9)$ \\
2001 & 85 & $49(58)$ & 429 & $96(22)$ \\
2002 & 80 & $49(61)$ & 537 & $91(17)$ \\
Total & 449 & $232(52)$ & 3,053 & $440(14)$ \\
\hline
\end{tabular}

\footnotetext{
I: Capnocytophaga
}

2: Number of children who were Capnocytophaga spp. carriers at least once during the studied period. 


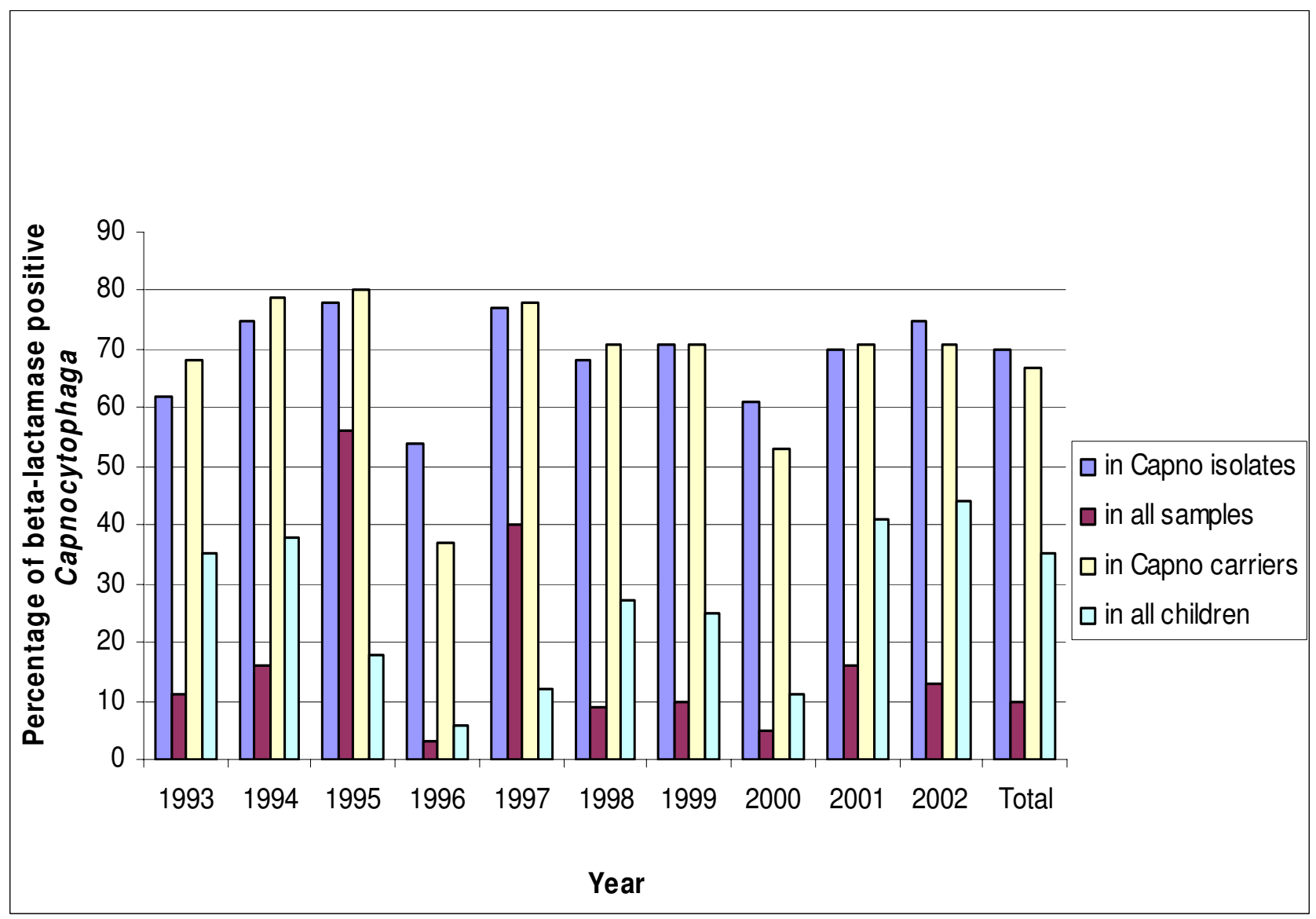

\section{Figure I}

Prevalence of beta-lactamase producing Capnocytophaga spp. strains in periodic oral samples from children hospitalized in the Pediatric Oncology Department of Hôpital Sud (Rennes, France) from 1993 to 2002.

Whatever the number of isolates, there was a high prevalence of beta-lactamase-producing strains $(70 \%)$, with a varying incidence from 54\% in 1996 (based on three out of 49 children) to $78 \%$ in 1995 (similarly, 11/14 isolates describes 8/45 children) (Figures 1 and 2). The susceptibility of Capnocytophaga strains was always conserved with imipenem (MIC $<4 \mu \mathrm{g} / \mathrm{ml}$ ) and amoxicillin combined with clavulanic acid (MIC $<4 / 2 \mu \mathrm{g} / \mathrm{ml}$ ). All beta-lactamase producing strains were uniformly resistant to cefazolin (MIC > $8 \mu \mathrm{g} / \mathrm{ml}$ ), but the authors noted different levels of resistance to amoxicillin and ceftazidime (Table 2).

\section{Discussion}

In immunocompromised children, a periodic survey of the oral cavity during hospitalization allows a predictive evaluation of the risk of systemic infections by Capnocytophaga spp.
In this study, the authors correlated the number of Capnocytophaga isolates with the number of child carriers of Capnocytophaga, indicating that protocol was correctly conducted. A decrease in incidence was observed during the 1995-1997 period, which could be due to use, in firstline antibiotic protocols, of beta-lactamase inhibitor combinations. During the other time periods, extended-spectrum antibiotic were given. Aminoglycosides were always associated to beta-lactam antibiotics in all protocols from 1993 to 2002. Incidence rates of total extended-spectrum beta-lactamase producing bacteria (ESBL) in gram-negative rods responsible for infection in Hôpital Sud (Rennes, France), calculated for 1,000 days of hospitalization, varied in 2002 from 0.04 to 0.7 depending on the department (higher rate in reanimation units) http:// www.cclinouest.comhttp://www.cclinouest.com/PDF/ r bmr2002 internet.pdf. Interestingly, all Capnocytophaga 


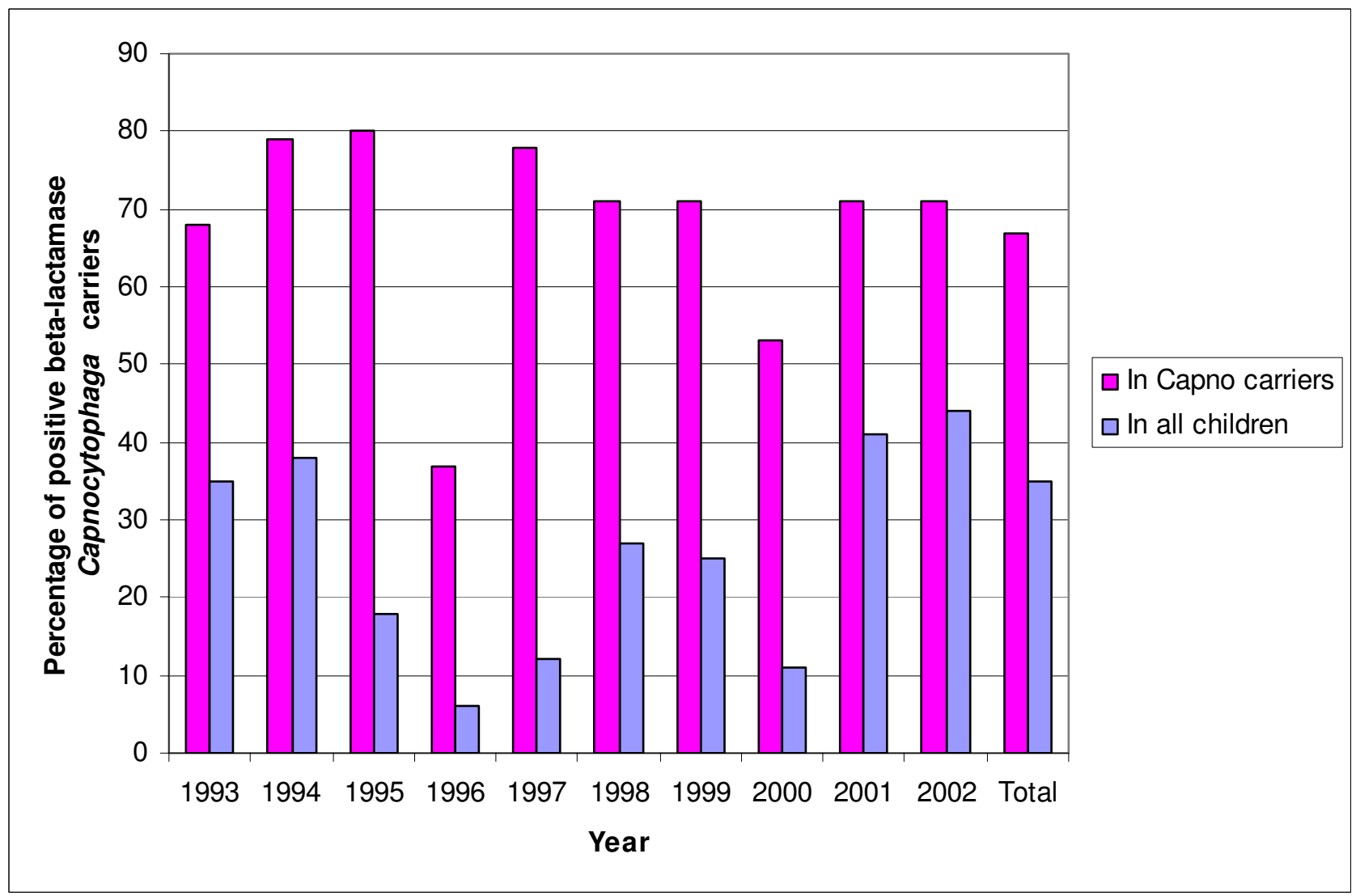

\section{Figure 2}

Percentage of positive beta-lactamase Capnocytophaga spp. carriers, from children hospitalized in the Pediatric Oncology Department of Hôpital Sud (Rennes, France) from 1993 to 2002.

spp. strains collected in this study appeared as colonizing strains, because neither bacteremia, nor other systemic infections, were observed during this study. Results of MIC determinations agree with previous works reporting that beta-lactamases confer a high degree of resistance to a wide range of beta-lactam antibiotics [14] while having no effect on imipenem and beta-lactamase-inhibitor combinations $[15,16]$. In a Canadian study, Roscoe et al. [17] reported that $36 \%$ of the strains collected mainly from clinical sources were beta-lactamase producers, while in a study in Taiwan, Lin et al. [3] reported that $18 \%$ of Capnocytophaga strains isolated from patients with bacteremia were beta-lactam resistant. In our study, the prevalence of beta-lactamase producers did not increase linearly during the study period.
A first hypothesis to explain these changes in incidence of beta-lactamase production could be the impact of current and previous therapy especially antimicrobial treatments or pathology, on the oral carriage of Capnocytophaga (study in progress). In a previous French study, Maury et al. [18] reported a high prevalence of beta-lactamase-producing Capnocytophaga species $(75 \%)$, which they associated with previous beta-lactam treatments. Even if Capnocytophaga spp. belongs to the so-called late colonizers in the normal flora, meaning that it is more frequently found in children at 12 months and later, in a follow-up study of healthy infants from the age of 2 to 12 months, [19] Nyfors et al. reported a positive correlation between antimicrobial exposure and beta-lactamase production in oral anaerobic gram-negative species, while reporting 
Table 2: Susceptibility of oral Capnocytophaga spp. isolated from 1993 to 2002 (Hôpital Sud, Rennes, France). Susceptibility testing was determined by standard methods and break points according to the criteria of NCCLS [13] and Bremmelgaard et al. [12]

\begin{tabular}{|c|c|c|}
\hline \multirow[t]{2}{*}{ Year } & \multicolumn{2}{|c|}{$\begin{array}{c}\text { Number of Resistant or Intermediate Capnocytophaga strains (\%) (Total number of } \\
\text { Capno strains }=440 \text { ) }\end{array}$} \\
\hline & Amoxicillin $\mathrm{MIC}^{\prime}>4 \mu \mathrm{g} / \mathrm{ml}$ & Ceftazidime MIC $>4 \mu \mathrm{g} / \mathrm{ml}$ \\
\hline 1993 & $31(88)$ & $28(80)$ \\
\hline 1994 & $31(84)$ & $28(75)$ \\
\hline 1995 & II (100) & $11(100)$ \\
\hline 1996 & $6(100)$ & $5(83)$ \\
\hline 1997 & $10(100)$ & $9(90)$ \\
\hline 1998 & $27(96)$ & 20 (7I) \\
\hline 1999 & $23(85)$ & $10(37)$ \\
\hline 2000 & $16(84)$ & $12(63)$ \\
\hline 2001 & $54(80)$ & $38(56)$ \\
\hline 2002 & $56(81)$ & $34(49)$ \\
\hline Total & $265(86)$ & $195(63)$ \\
\hline
\end{tabular}

Note: No strains were resistant to an imipenem and amoxicillin/clavulanic acid combination, and all beta-lactamase-producing strains were resistant to cefazolin.

I: Minimal Inhibitory Concentration $(\mu \mathrm{g} / \mathrm{ml})$.

only two beta-lactamase-producing C. ochracea isolates. In a recent contradictory study, [20]Capnocytophaga spp. has been detected in $1.9 \%$ of all episodes of fever and neutropenia before antibiotic therapy, versus $0.3 \%$ during antimicrobial treatment.

A second hypothesis to explain these changes in incidence of beta-lactamase production could be linked to the immediate environment and close contact. These conditions have a great influence on the composition of the flora, and coming and going between home and hospital could modify the children's oral ecosystem. A turnover of the bacterial population from beta-lactamase-positive to beta-lactamase-negative strains may occur in young children with a developing oral ecosystem.

Another explanation of this high prevalence of beta-lactamase production could be conferred on transfer of encoding-resistance genes. Anaerobic bacteria are known to be able to exchange genetic material with aerobic bacteria, even though antibiotic-resistance genes are expressed differently in aerobic and anaerobic bacteria [21]. The spread of antibiotic resistance can also play a great part in nosocomial infections in neutropenic patients [22]. The high rate of resistance to third-generation cephalosporins observed in this study could be due to the dissemination of an epidemic clone. Some ceftazidime-resistant strains have the same susceptibility and plasmid profiles (data not shown). Other authors have described resistant clinical isolates $[5,6,14,16]$, but Rosenau et al. [8] were the first to characterize a plasmid-encoded TEM extended-spectrum beta-lactamase in Capnocytophaga spp. Clavulanate- sensitive cephalosporinases belonging to class A group $2 \mathrm{e}$ from the classification of Bush et al. [23] have recently been described $[7,24]$. They differ by only one or two substitutions in their sequences ( $c f x \mathrm{~A}$, Genbank accession No. U75371; cfxA2, Genbank accession No. AF118110; cfxA3, Genbank accession No. AF472622). Large transposons encode all the functions needed for their own conjugation and for resistance to antimicrobial agents, including thirdgeneration cephalosporins.

\section{Conclusion}

These results show the importance of testing for the antibiotic susceptibility of and beta-lactamase production by clinical Capnocytophaga strains, in which beta-lactamase production has become very common. In neutropenic patients, resistance to third-generation cephalosporins should be taken into consideration upon hospitalization to adapt the empiric antimicrobial treatment previously dispensed.

\section{Competing interests}

The author(s) declare that they have no competing interests.

\section{Authors' contributions}

AJG carried out the microbiological studies and wrote the manuscript. ZTS, LD and NMG participated in the microbiological studies. VG was in charge of the clinical studies. Provision of advice was given by JLS, MC and MBM. All authors read and approved the final manuscript. 


\section{Acknowledgements}

We would like to thank Gene Bourgeau and Céline Allaire for editorial assistance.

This work was supported by the Fondation Langlois and Conseil Régional de Bretagne.

\section{References}

I. Nonnenmacher C, Mutters R, de Jacoby LF: Microbiological characteristics of subgingival microbiota in adult periodontitis, localized juvenile periodontitis and rapidly progressive periodontitis subjects. Clin Microbiol Infect 200I, 7(4):2 I3-2I 7 .

2. Buu-Hoi AY, Joundy S, Acar JF: Endocarditis caused by Capnocytophaga ochracea. J Clin Microbiol 1988, 26(5): I 06 I- 1062.

3. Lin RD, Hsueh PR, Chang SC, Luh KT: Capnocytophaga bacteremia: clinical features of patients and antimicrobial susceptibility of isolates. J Formos Med Assoc 1998, 97( (1):44-48.

4. Parenti DM, Snydman DR: Capnocytophaga species: infections in nonimmunocompromised and immunocompromised hosts. J Infect Dis 1985, I 5 I: | $40-147$.

5. Arlet G, Sanson-Le Pors MJ, Casin IM, Ortenberg M, Perol Y: In vitro susceptibility of 96 Capnocytophaga strains, including a betalactamase producer, to new beta-lactam antibiotics and six quinolones. Antimicrob Agents Chemother I987, 3 I (8): | 283- I 284.

6. Gomez-Garces JL, Alos JI, Sanchez J, Cogollos R: Bacteremia by multidrug-resistant Capnocytophaga sputigena. J Clin Microbiol 1994, 32: 1067-1069.

7. Jolivet-Gougeon A, Tamanai-Shacoori Z, Desbordes L, Burggraeve N, Cormier M, Bonnaure-Mallet $M$ : Genetic analysis of an ambler class $A$ extended-spectrum beta-lactamase from Capnocytophaga ochracea. J Clin Microbiol 2004, 42(2):888-890.

8. Rosenau A, Cattier B, Gousset N, Harriau P, Philippon A, Quentin R: Capnocytophaga ochracea: characterization of a plasmidencoded extended-spectrum TEM-I 7 beta-lactamase in the phylum Flavobacter-bacteroides. Antimicrob Agents Chemother 2000, 44(3):760-762.

9. Bonnaure-Mallet M, Bunetel L, Tricot-Doleux S, Guerin J, Bergeron $C$, LeGall E: Oral complications during treatment of malignant diseases in childhood: effects of tooth brushing. Eur J Cancer 1998, 34(10): 1588-159|.

10. Mashimo PA, Yamamoto Y, Nakamura M, Slots J: Selective recovery of oral Capnocytophaga spp. with sheep blood agar containing bacitracin and polymixin B. J Clin Microbiol 1983, I 7:|87-191.

II. Slots ]: Enzymatic characterization of some oral and nonoral gram-negative bacteria with the API ZYM system. J Clin Microbiol 1981, I4:288-294.

12. Bremmelgaard A, Pers C, Kristiansen JE, Korner B, Heltberg O, Frederiksen W: Susceptibility testing of Danish isolates of Capnocytophaga and CDC group DF-2 bacteria. APMIS 1989, 97(I):43-48.

13. NCCLS: Methods for antimicrobial susceptibility testing of anaerobic bacteria: approved standard. 4th edition edition. Wayne, NCCLS; 1997:MII-AI4.

14. Jolivet-Gougeon A, Buffet A, Dupuy C, Sixou JL, Bonnaure-Mallet M, David S, Cormier M: In vitro susceptibilities of Capnocytophaga isolates to beta-lactam antibiotics and beta-lactamase inhibitors. Antimicrob Agents Chemother 2000, 44(II):3186-3188.

15. Rummens JL, Gordts B, Van Landuyt HW: In vitro susceptibility of Capnocytophaga species to 29 antimicrobial agents. Antimicrob Agents Chemother 1986, 30(5):739-742.

16. Foweraker JE, Hawkey PM, Heritage J, Van Landuyt HW: Novel beta-lactamase from Capnocytophaga sp. Antimicrob Agents Chemother 1990, 34(8): I50|-I504.

17. Roscoe DL, Zemcov SJ, Thornber D, Wise R, Clarke AM: Antimicrobial susceptibilities and beta-lactamase characterization of Capnocytophaga species. Antimicrob Agents Chemother 1992, 36(10):2197-2200.

18. Maury S, Leblanc T, Rousselot P, Legrand P, Arlet G, Cordonnier C: Bacteremia due to Capnocytophaga species in patients with neutropenia: high freqency of b-lactamase-producing strains. Clin Infect Dis 1999, 28(4): I I72-I I 74.
19. Nyfors S, Kononen E, Takala A, Jousimies-Somer H: Beta-lactamase production by oral anaerobic gram-negative species in infants in relation to previous antimicrobial therapy. Antimicrob Agents Chemother 1999, 43(7): I59I-I594.

20. Ammann RA, Hirt A, Luthy AR, Aebi C: Predicting bacteremia in children with fever and chemotherapy-induced neutropenia. Pediatr Infect Dis J 2004, 23(I):6I-67.

21. Guiney DG, Hasegawa $P$, Davis CE: Plasmid transfer from Escherichia coli to Bacteroides fragilis: differential expression of antibiotic resistance phenotypes. Proc Natl Acad Sci USA 1984, 8 I (22):7203-7206.

22. Girlich D, Poirel L, Leelaporn A, Karim A, Tribuddharat C, Fennewald $M$, Nordmann P: Molecular epidemiology of the integronlocated VEB-I extended-spectrum beta-lactamase in nosocomial enterobacterial isolates in Bangkok, Thailand. J Clin Microbiol 200I, 39(I): I75-182.

23. Bush K, Jacoby GA, Medeiros AA: A functional classification scheme for beta-lactamases and its correlation with molecular structure. Antimicrob Agents Chemother 1995, 39(6): I 21 I - I 233.

24. Parker AC, Smith Cl: Genetic and biochemical analysis of a novel Ambler class A beta-lactamase responsible for cefoxitin resistance in Bacteroides species. Antimicrob Agents Chemother 1993, 37(5):1028-1036.

\section{Pre-publication history}

The pre-publication history for this paper can be accessed here:

http://www.biomedcentral.com/1471-2334/5/32/prepub
Publish with Biomed Central and every scientist can read your work free of charge

"BioMed Central will be the most significant development for disseminating the results of biomedical research in our lifetime. "

Sir Paul Nurse, Cancer Research UK

Your research papers will be:

- available free of charge to the entire biomedical community

- peer reviewed and published immediately upon acceptance

- cited in PubMed and archived on PubMed Central

- yours - you keep the copyright

Submit your manuscript here:

http://www.biomedcentral.com/info/publishing_adv.asp
BioMedcentral 